\title{
When is Quantum Decoherence Dynamics Classical?
}

\author{
Jiangbin Gong and Paul Brumer \\ Chemical Physics Theory Group, Department of Chemistry, \\ University of Toronto, Toronto, Canada M5S $3 H 6$
}

(Dated: May 6, 2019)

\begin{abstract}
A direct classical analog of quantum decoherence is introduced. Similarities and differences between decoherence dynamics examined quantum mechanically and classically are exposed via a second-order perturbative treatment and via a strong decoherence theory, showing a strong dependence on the nature of the system-environment coupling. For example, for the traditionally assumed linear coupling, the classical and quantum results are shown to be in exact agreement.
\end{abstract}

PACS numbers: 03.65.Yz

Decoherence is the loss of quantum coherence due to system-bath coupling. There has been considerable theoretical and experimental work demonstrating that quantum-classical correspondence (QCC) can be induced by decoherence [1, 2, 3]. By contrast, little work has been done on examining the correspondence between classical and quantum descriptions of the time evolution of decoherence itself, i.e. decoherence dynamics. In this Letter we show that (a) one can introduce a direct classical analog of quantum decoherence, and (b) by examining the dynamics of decoherence classically one gains new insights into both the dynamics of decoherence described quantum mechanically and into the conditions for QCC of the dynamics of decoherence. For example, we show that the extent of QCC depends strongly on the nature of the system-bath coupling and far less upon $\hbar$ than expected, that results assumed to be quantum mechanical can be obtained classically and that nonlinear system-bath coupling can cause nonclassical decoherence dynamics even for macroscopic systems.

The formal Liouville-based theory of QCC in an isolated system 国 makes clear that there is a strict analogy between quantum and classical dynamics in phase space. As in the quantum case, the classical Liouville dynamics of a closed system is unitary and we expect the reduced classical Liouville dynamics of a system coupled to a bath to be nonunitary. We therefore suspect that, due to bath's coarse-graining effects, the reduced dynamics of the system propagated classically will show decoherence dynamics that is, qualitatively, parallel to that seen in quantum dynamics insofar as the loss of phase information, entropy production, etc.

Here we quantitatively compare the dynamics of decoherence that is induced quantum mechanically to that induced classically. This is done by analytically examining the dynamics of an initial quantum state, in a system coupled to a bath, that is propagated either quantum mechanically or classically. The observed (rather remarkable) similarities and differences between the classical and quantum decoherence dynamics should be of considerable interest to a variety of modern fields such as quantum information processing [5] and quantum control of atomic and molecular processes [6]. Further, this analysis offers new insights into both decoherence and QCC, is relevant to semiclassical descriptions of decoherence dynamics [7], and has motivated purely classical descriptions of dynamics-induced intrinsic decoherence, with preliminary computations [8] that support the analytic results presented here.

We begin by introducing classical analogs of some representation-independent and representation-dependent measures of decoherence. A second-order perturbative treatment is then used to examine QCC in early-time decoherence dynamics. Subsequently, we introduce a classical theory of strong decoherence that allows us to go beyond perturbation theory; the results are then compared with corresponding quantum theory.

Consider a system with a time-independent Hamiltonian $H^{s}=P^{2} /(2 m)+V(Q)$, where $(Q, P)$ are conjugate position and momentum variables, coupled to $N$ independent harmonic bath modes described by the Hamiltonians $H_{j}^{b}=p_{j}^{2} /\left(2 m_{j}\right)+m_{j} \omega_{j}^{2} q_{j}^{2} / 2$, where $\left\{p_{j}, q_{j}\right\}(j=1,2, \cdots, N)$ are bath-mode phase space variables. The systembath coupling potential is assumed to be $V^{s b}=\sum_{j=1}^{N} C_{j} f(Q) q_{j}$ so that the total Hamiltonian is given by $H=$ $H^{s}+\sum_{j=1}^{N}\left[H_{j}^{b}+C_{j} f(Q) q_{j}\right]$. The phase space distribution function evolved classically and the quantum Wigner function for the entire phase space are represented by $\rho^{c}\left[Q, P,\left\{q_{j}, p_{j}\right\}, t\right]$ and $\rho^{W}\left[Q, P,\left\{q_{j}, p_{j}\right\}, t\right]$, respectively. Their time evolution is given by $\partial \rho^{c} / \partial t=\left\{H, \rho^{c}\right\}_{P}$ and $\partial \rho^{W} / \partial t=\left\{H, \rho^{W}\right\}_{M}$, where $\{\cdot\}_{P}$ denotes classical Poisson bracket and $\{\cdot\}_{M}$ denotes quantum Moyal bracket. Further, we define classical and quantum reduced distribution functions $\tilde{\rho}_{c}(Q, P, t) \equiv \int \rho^{c} d \Gamma_{b}^{N}$ and $\tilde{\rho}_{W}(Q, P, t) \equiv \int \rho^{W} d \Gamma_{b}^{N}$, where $d \Gamma_{b}^{N} \equiv \prod_{j=1}^{N} d q_{j} d p_{j}$. Our interest is in the correspondence between the classical and quantum decoherence dynamics of initial states that can be either classical (i.e. positive Wigner density everywhere) or nonclassical [ $\left[\right.$ (e.g. displaying regions of negative $\rho^{W}\left[Q, P,\left\{q_{j}, p_{j}\right\}, t\right]$ ).

Consider two measures of decoherence in each of classical and quantum mechanics. One widely-used and representation-independent measure is the linear entropy $S_{q} \equiv 1-\operatorname{Tr}\left(\hat{\tilde{\rho}}^{2}\right)$ [9], where $\hat{\tilde{\rho}}$ is the reduced density operator of the system. An increase in $S_{q}$ causes $1 /\left(1-S_{q}\right)$ to increase, corresponding to an increasing number of incoherently 
populated orthogonal quantum states. Since $S_{q}=1-2 \pi \hbar \int \tilde{\rho}_{W}^{2}(Q, P, t) d \Gamma_{s}$, where $d \Gamma_{s} \equiv d Q d P$, this entropy has a natural classical analog (denoted $S_{c}$ ) obtained by replacing $\tilde{\rho}_{W}$ with $\tilde{\rho}_{c}$. That is, $S_{c} \equiv 1-2 \pi \hbar \int \tilde{\rho}_{c}^{2}(Q, P, t) d \Gamma_{s}$. A more detailed, but representation-dependent description of decoherence is the decay of off-diagonal density matrix elements such as $\left\langle Q_{1}|\hat{\tilde{\rho}}(t)| Q_{2}\right\rangle$. Significantly, we discover that the classical analog of these matrix elements can also be constructed. Specifically, noting that $\left\langle Q_{1}|\hat{\tilde{\rho}}(t)| Q_{2}\right\rangle=\int d P \tilde{\rho}_{W}(\bar{Q}, P, t) \exp [i \Delta Q P / \hbar]$, where $\bar{Q} \equiv\left(Q_{1}+Q_{2}\right) / 2$ and $\Delta Q=Q_{1}-Q_{2}$, we define the classical analog (denoted $\tilde{\rho}_{c}\left(Q_{1}, Q_{2}, t\right)$ ) of $\left\langle Q_{1}|\hat{\tilde{\rho}}(t)| Q_{2}\right\rangle$ as the Fourier transformed classical distribution function, i.e., $\tilde{\rho}_{c}\left(Q_{1}, Q_{2}, t\right) \equiv \int d P \tilde{\rho}_{c}(\bar{Q}, P, t) \exp [i \Delta Q P / \hbar]$. This approach can be readily extended to the momentum representation.

Perturbative treatments have proved to very useful in understanding decoherence dynamics [10, 11. Here, to examine classical vs. quantum decoherence dynamics at short times, a regime of great interest in the control of decoherence, we consider a second-order expansion with respect to time variable $t$ for both $S_{c}$ and $S_{q}$, i.e., $S_{c}(t)=$ $S_{c}(0)+t / \tau_{c, 1}+t^{2} / \tau_{c, 2}^{2}+\cdots$, and $S_{q}(t)=S_{q}(0)+t / \tau_{q, 1}+t^{2} / \tau_{q, 2}^{2}+\cdots$. Using the definitions of Poisson and Moyal brackets and assuming that the initial distribution function is decorrelated with initial bath statistics, we obtain

$$
\begin{gathered}
\frac{1}{\tau_{c, 1}}=\frac{1}{\tau_{q, 1}}=0, \\
\frac{1}{\tau_{c, 2}^{2}}=\frac{C_{b}}{\hbar} \int d Q_{1} d Q_{2}\left|\tilde{\rho}_{c}\left(Q_{1}, Q_{2}, 0\right)\right|^{2} \Delta Q^{2}\left[\frac{d f(\bar{Q})}{d \bar{Q}}\right]^{2},
\end{gathered}
$$

and

$$
\frac{1}{\tau_{q, 2}^{2}}=\frac{C_{b}}{\hbar} \int d Q_{1} d Q_{2}\left|\left\langle Q_{1}|\hat{\tilde{\rho}}(0)| Q_{2}\right\rangle\right|^{2} \Delta Q^{2}\left[\frac{\Delta f(\bar{Q})}{\Delta Q}\right]^{2},
$$

where $C_{b}=\sum_{j=1}^{N} C_{j}^{2} \operatorname{coth}\left(\beta \hbar \omega_{j} / 2\right) /\left(2 m_{j} \omega_{j}\right), \Delta f(\bar{Q}) \equiv f(\bar{Q}+\Delta Q / 2)-f(\bar{Q}-\Delta Q / 2)$, and $\beta$ is the Boltzmann factor. Note that the factor $\hbar$ appearing in the classical result [Eq. 2)] is just due to the definitions of $S_{c}$ and $\tilde{\rho}_{c}\left(Q_{1}, Q_{2}, 0\right)$, and that the initial variances of the bath variables $q_{j}$ have been evaluated using quantum statistics to ensure the same initial quantum state for the ensuing classical and quantum dynamics. Note also that the decoherence time scale indicated in the easily-derived and simple quantum result of Eq. (3) is consistent with, but is more transparent than, a previous perturbation result (Eq. (5.6) in Ref. 12) obtained using a sophisticated influence functional approach.

Equation (1) shows that zero first-order decoherence rate i.e., $1 / \tau_{q, 1}=0$, has a strict classical analog. More interestingly, Eqs. (2) and (3) show that, for the same fixed initial distribution function, the ratio of $1 / \tau_{q, 2}^{2}$ to $1 / \tau_{c, 2}^{2}$ is $\hbar$-independent. As seen from Eqs. (2) and (3), $\left(1 / \tau_{q, 2}^{2}-1 / \tau_{c, 2}^{2}\right)$ arises from the difference between the derivative $d f / d Q$ and the finite-difference function $\Delta f / \Delta Q$, weighted by $\Delta Q^{2}$ and the initial state. As a result: (1) For any given $f(Q)$, as long as $\left\langle Q_{1}|\hat{\tilde{\rho}}(0)| Q_{2}\right\rangle$ decays fast enough with $|\Delta Q|$ such that $\Delta f / \Delta Q \approx d f / d Q$, there would be excellent QCC in early-time decoherence dynamics. The smaller the $\hbar$, the more rigorous is this requirement. (2) If $f(Q)$ depends only linearly or quadratically upon the coupling coordinate $Q$, then $\left(1 / \tau_{q, 2}^{2}-1 / \tau_{c, 2}^{2}\right)=0$ for any initial state. Significantly then, in all traditional decoherence models 13] where $f(Q)=Q$ is assumed, there exists perfect QCC in early decoherence dynamics, regardless of $\hbar$, and irrespective of the system potential $V(Q)$ 14. Indeed, in the case of $f(Q)=Q$ Eq. (2) reduces to an important result, previously obtained quantum mechanically 10:

$$
\frac{1}{\tau_{c, 2}^{2}}=\frac{1}{\tau_{q, 2}^{2}}=2 \frac{\delta^{2} Q}{\hbar} \sum_{j=1}^{N} \frac{C_{j}^{2}}{2 m_{j} \omega_{j}} \operatorname{coth}\left(\frac{\beta \hbar \omega_{j}}{2}\right) .
$$

where the initial state of the system is assumed to be pure, with the initial variance in $Q$ given by $\delta^{2} Q$. (3) For nonlinear $f(Q)$ where $\Delta f / \Delta Q \neq d f / d Q$ over the range of the initial state, QCC can be very poor.

The second-order perturbative treatment is most reliable at short times and for weak decoherence. The results are particularly significant for studies of decoherence control where early-time dynamics of weak decoherence is important. In these circumstances it is useful to understand the extent to which (quantum) decoherence is equivalent to classical entropy production, i.e. to increasing $S_{c}(t)$. In particular, if there exists good correspondence between classical and quantum decoherence dynamics, then the essence of decoherence control is equivalent to the suppression of classical entropy production, and various classical tools may be considered to achieve decoherence control. If not, then fully quantum tools are required.

As an example, consider decoherence for an initial superposition state of two well-separated and strongly localized Gaussian wavepackets located at $Q_{a}=\bar{Q}_{a b}-\Delta Q_{a b} / 2$ and $Q_{b}=\bar{Q}_{a b}+\Delta Q_{a b} / 2$ with $\bar{Q}_{a b}=0$. For this initial state, 
$1 / \tau_{c, 2}^{2} \sim\left(C_{b} / \hbar\right) \Delta Q_{a b}^{2}\left[d f\left(\bar{Q}_{a b}\right) / d \bar{Q}_{a b}\right]^{2}$, and $1 / \tau_{q, 2}^{2} \sim\left(C_{b} / \hbar\right) \Delta Q_{a b}^{2}\left[\Delta f\left(\bar{Q}_{a b}\right) / \Delta Q_{a b}\right]^{2}$. Then in a cubic decoherence model, for example, where $f(Q)=Q^{3}$, one would obtain $1 / \tau_{c, 2}^{2} \sim 0$ since $d f\left(\bar{Q}_{a b}\right) / d \bar{Q}_{a b}=0$. However, here $1 / \tau_{q, 2}^{2}>>1 / \tau_{c, 2}^{2}$, i.e. there is appreciable decoherence without classical entropy production. By contrast, in another nonlinear decoherence model where $f(Q)=\sin \left(2 \pi Q / \Delta Q_{a b}+\pi / 4\right), 1 / \tau_{q, 2}^{2} \sim 0$ since $f\left(Q_{a}\right)=f\left(Q_{b}\right)$. Here, however, $1 / \tau_{c, 2}^{2}>>1 / \tau_{q, 2}^{2}$, i.e., the system is decoherence-free but with substantial classical entropy production. Since we find that the ratio of $\tau_{q, 2}^{2}$ to $\tau_{c, 2}^{2}$ in early-time decoherence dynamics is independent of $\hbar$ for fixed initial state, these two examples lead to a rather counter-intuitive result: given a macroscopic object which is initially in a superposition state of two distinguishable states and is nonlinearly coupled with an environment, classical dynamics could totally fail to predict its initial entropy production or its decoherence rate. Indeed, Eqs. (2) and (3) suggest that, as long as $d f(\bar{Q}) / d \bar{Q} \neq 0$ and $|f(Q)|$ is bounded, then $1 / \tau_{q, 2}^{2}$ saturates with increasing $\Delta Q_{a b}$, whereas $1 / \tau_{c, 2}^{2}$ does not. Thus, one can conclude that decoherence dynamics must be quantum and that the system-environment coupling must be nonlinear if the saturation behavior of early-time decoherence rates is observed experimentally[15. Further, it is clear that in the limit of large $\Delta Q_{a b}$, classical decoherence dynamics in the general case of nonlinear systemenvironment coupling predicts much faster decoherence than does quantum decoherence dynamics. This leads to the rather surprising inference that initial superposition states of well-separated wavepackets would be more susceptible to nonlinear system-environment coupling if they are propagated by classical dynamics than by quantum mechanics.

To go beyond the perturbation results we now consider a strong decoherence model in which decoherence is assumed to be much faster than the system dynamics, so that $H^{s}$ can be set to zero [1]. We consider both the "off-diagonal elements" $\tilde{\rho}_{c}\left(Q_{1}, Q_{2}, t\right)$ as well as the entropy $S_{c}(t)$ and compare them to the quantum results.

In this case the classical Liouville dynamics gives

$$
\begin{aligned}
& \frac{\partial F_{c}\left[\bar{Q}, \Delta Q,\left\{q_{j}, p_{j}\right\}, t\right]}{\partial t}=\sum_{k=1}^{N} \frac{\partial H_{k}^{b}}{\partial q_{k}} \frac{\partial F_{c}\left[\bar{Q}, \Delta Q,\left\{q_{j}, p_{j}\right\}, t\right]}{\partial p_{k}} \\
& -\sum_{k=1}^{N} \frac{\partial H_{k}^{b}}{\partial p_{k}} \frac{\partial F_{c}\left[\bar{Q}, \Delta Q,\left\{q_{j}, p_{j}\right\}, t\right]}{\partial q_{k}}+\sum_{k=1}^{N} C_{k} f(\bar{Q}) \frac{\partial F_{c}\left[\bar{Q}, \Delta Q,\left\{q_{j}, p_{j}\right\}, t\right]}{\partial p_{k}} \\
& -\frac{i}{\hbar} \Delta Q \sum_{k=1}^{N} C_{k} \frac{d f(\bar{Q})}{d \bar{Q}} q_{k} F_{c}\left[\bar{Q}, \Delta Q,\left\{q_{j}, p_{j}\right\}, t\right],
\end{aligned}
$$

where $F_{c}\left(\bar{Q}, \Delta Q,\left\{q_{j}, p_{j}\right\}, t\right) \equiv \int d P \exp [i \Delta Q P / \hbar] \rho_{c}\left[\bar{Q}, P,\left\{q_{j}, p_{j}\right\}, t\right]$. Since $\dot{Q}=0$ due to $H_{s}=0$, and $\Delta Q$ is a time-independent parameter introduced in the Fourier transformation, Eq. (5) leads to

$$
\begin{aligned}
& \frac{d F_{c}\left[\bar{Q}, \Delta Q,\left\{q_{j}(t), p_{j}(t)\right\}, t\right]}{d t}=\frac{\partial F_{c}\left[\bar{Q}, \Delta Q,\left\{q_{j}(t), p_{j}(t)\right\}, t\right]}{\partial t} \\
& +\sum_{k=1}^{N} \frac{\partial F_{c}\left[\bar{Q}, \Delta Q,\left\{q_{k}(t), p_{k}(t)\right\}, t\right]}{\partial q_{k}(t)} \dot{q}_{k}(t)+\sum_{k=1}^{N} \frac{\partial F_{c}\left[\bar{Q}, \Delta Q,\left\{q_{k}(t), p_{k}(t)\right\}, t\right]}{\partial p_{k}(t)} \dot{p}_{k}(t) \\
& =-\frac{i}{\hbar} \Delta Q \sum_{k=1}^{N} C_{k} q_{k}(t) \frac{d f(\bar{Q})}{d \bar{Q}} F_{c}\left[\bar{Q}, \Delta Q,\left\{q_{j}(t), p_{j}(t)\right\}, t\right]
\end{aligned}
$$

where $\left\{q_{j}(t), p_{j}(t)\right\}$ satisfy $\dot{q}_{j}(t)=\partial H_{j}^{b} / \partial p_{j}(t)$ and $\dot{p}_{j}(t)=-\partial H_{j}^{b} / \partial q_{j}(t)-C_{j} f(\bar{Q})$, of which the solution is

$$
q_{j}(t)=\frac{C_{j} f(\bar{Q})}{m_{j} \omega_{j}^{2}}\left[\cos \left(\omega_{j} t\right)-1\right]+q_{j}(0) \cos \left(\omega_{j} t\right)+\frac{p_{j}(0)}{m_{j} \omega_{j}} \sin \left(\omega_{j} t\right),
$$

and $p_{j}(t)=m_{j} \dot{q}_{j}(t)$. Analytically integrating Eq. (6), and using $d \Gamma_{b}^{N}(t)=d \Gamma_{b}^{N}(0)$ and $\tilde{\rho}_{c}\left(Q_{1}, Q_{2}, t\right)=$ $\int d \Gamma_{b}^{N}(t) F_{c}\left[\bar{Q}, \Delta Q,\left\{q_{j}(t), p_{j}(t)\right\}, t\right]$, we have

$$
\begin{aligned}
& \tilde{\rho}_{c}\left(Q_{1}, Q_{2}, t\right)=\int d \Gamma_{b}^{N}(0) F_{c}\left[\bar{Q}, \Delta Q,\left\{q_{j}(0), p_{j}(0)\right\}, 0\right] \\
& \times \exp \left[-\frac{i}{\hbar} \int_{0}^{t} d t \Delta Q \sum_{k=1}^{N} C_{k} \frac{d f(\bar{Q})}{d \bar{Q}} q_{k}(t)\right]
\end{aligned}
$$

Substituting Eq. (7) into Eq. (8), using the initial quantum state of the bath that is initially uncorrelated with the 
system, and assuming that the equilibrium state of the bath is maintained, we obtain

$$
\frac{\tilde{\rho}_{c}\left(Q_{1}, Q_{2}, t\right)}{\tilde{\rho}_{c}\left(Q_{1}, Q_{2}, 0\right)}=\exp \left[i \phi_{c}(t)-(\Delta Q)^{2}\left(\frac{d f(\bar{Q})}{d \bar{Q}}\right)^{2} B_{2}(t)\right],
$$

where $\phi_{c}(t) \equiv(\Delta Q) f(\bar{Q})[d f(\bar{Q}) / d \bar{Q}] B_{1}(t) / \hbar$, with $B_{1}(t)=\sum_{j=1}^{N} C_{j}^{2}\left[t-\sin \left(\omega_{j} t\right) / \omega_{j}\right] /\left(m_{j} \omega_{j}^{2}\right)$, and $B_{2}(t)=$ $\sum_{j=1}^{N} C_{j}^{2} \operatorname{coth}\left(\beta \hbar \omega_{j} / 2\right)\left[1-\cos \left(\omega_{j} t\right)\right] /\left(2 m_{j} \hbar \omega_{j}^{3}\right)$. Interestingly, the classical result [Eq. (9)] displays two dynamical aspects of $\tilde{\rho}_{c}\left(Q_{1}, Q_{2}, t\right)$, i.e., coherent dynamics of its phase $\phi_{c}(t)$, and incoherent decay due to bath statistics. The classical linear entropy $S_{c}(t)$ can then be obtained from Eq. (9) as

$$
S_{c}(t)=1-\int d Q_{1} d Q_{2}\left|\tilde{\rho}_{c}\left(Q_{1}, Q_{2}, 0\right)\right|^{2} \exp \left[-2(\Delta Q)^{2}\left(\frac{d f(\bar{Q})}{d \bar{Q}}\right)^{2} B_{2}(t)\right] .
$$

With similar manipulations for quantum strong decoherence dynamics, we obtain the quantum result

$$
\frac{\left\langle Q_{1}|\hat{\tilde{\rho}}(t)| Q_{2}\right\rangle}{\left\langle Q_{1}|\hat{\tilde{\rho}}(0)| Q_{2}\right\rangle}=\exp \left[i \phi_{q}(t)-\Delta Q^{2}\left(\frac{\Delta f(\bar{Q})}{\Delta Q}\right)^{2} B_{2}(t)\right]
$$

where $\left.\phi_{q}(t) \equiv \Delta Q f(\bar{Q})[\Delta f(\bar{Q}) / \Delta Q] B_{1}(t)\right] / \hbar$, and

$$
S_{q}(t)=1-\int d Q_{1} d Q_{2}\left|\left\langle Q_{1}|\hat{\tilde{\rho}}(0)| Q_{2}\right\rangle\right|^{2} \exp \left[-2(\Delta Q)^{2}\left(\frac{\Delta f(\bar{Q})}{\Delta Q}\right)^{2} B_{2}(t)\right] .
$$

These results extend those in Ref. [16] to nonlinear $f(Q)$ using a simple approach and demonstrate a direct classical analog to quantum strong decoherence dynamics.

Since $d B_{2}(t) / d t(t=0)=0$ and $d^{2} B_{2}(t) / d t^{2}(t=0)=C_{b} / \hbar$, one finds that in the short time limit, Eqs. (10) and (12) reduce to previous perturbation results of $1 / \tau_{c, 1}, 1 / \tau_{c, 2}^{2}, 1 / \tau_{q, 1}$, and $1 / \tau_{q, 2}^{2}$. Furthermore, the classical results [Eqs. (9) and (10)] are again much similar to the quantum results [Eqs. (11) and (12)], with the only difference being that $\Delta f / \Delta Q$ in the quantum expression is replaced by $d f / d Q$ in the classical result.

This result makes clear that our previous QCC results based upon second-order perturbation theory are generalizable to all orders of time in the strong decoherence case. In particular, defining $\gamma_{c}(t) \equiv$ $d \ln \left|\tilde{\rho}_{c}\left(Q_{1}, Q_{2}, t\right)\right| / d t$ and $\gamma_{q}(t) \equiv d \ln \left|\left\langle Q_{1}|\hat{\tilde{\rho}}(t)| Q_{2}\right\rangle\right| / d t$, we have $\gamma_{c}(t)=-(\Delta Q)^{2}[d f(\bar{Q}) / d \bar{Q}]^{2}\left(d B_{2}(t) / d t\right)$, and $\gamma_{q}(t)=-(\Delta Q)^{2}[\Delta f(\bar{Q}) / \Delta Q]^{2}\left(d B_{2}(t) / d t\right)$. Then, in the case of linear and/or quadratic coupling, e.g., $f(Q)=$ $a Q+b Q^{2}$, one has $\gamma_{c}(t)=\gamma_{q}(t)$ and $S_{c}(t)=S_{q}(t)$, showing that there is perfect QCC in decoherence dynamics for all times.

By contrast, in the case of nonlinear coupling, $\gamma_{c}(t)$ in general does not saturate with increasing $\Delta Q$ whereas $\gamma_{q}(t)$ does saturate for bounded $|f(Q)|$. As such, in the limit of large $\Delta Q$, one has $\left|\gamma_{c}(t)\right|>>\left|\gamma_{q}(t)\right|$ and thus $\left[1-S_{c}(t)\right]<<\left[1-S_{q}(t)\right]$ as $t$ increases, with $\left|\gamma_{c}(t) / \gamma_{q}(t)\right|$ independent of $\hbar$. This observation is of conceptual importance: it says that decoherence can dramatically improve QCC, but as far as some detailed characteristics of decoherence dynamics are concerned, decoherence itself does not necessarily suffice to ensure that the dynamics of quantum entropy production equals that of classical entropy production. That is, even in the presence of strong decoherence, subtle quantum classical differences may persist in some measures (e.g., $1 /\left[1-S_{q}(t)\right]$ vs. $\left.1 /\left[1-S_{c}(t)\right]\right)$ for all finite times. Note, however, the entropy measures such as $1 /\left[1-S_{q}(t)\right]$ are not a quantum mechanical observables and hence do not allow one to directly measure the subtle difference between classical and quantum decoherence dynamics at later times.

Thus, from both the perturbation and strong decoherence results, we obtain that QCC depends critically upon the initial quantum state and the nature of the system-environment coupling. This result should have an impact on our current understanding of decoherence even when the role of the dynamics of the system is important. For example, it is worthwhile reexamining the relationship between classical Lyapunov exponents and decoherence rates in classically chaotic systems, since previous studies [17] only dealt with the case of linear system-environment coupling.

In conclusion, we have examined, using analogous measures, the decoherence dynamics of an initial quantum state coupled to a bath that is subjected to either classical or quantum dynamics. Within the framework of a secondorder perturbative treatment and a strong decoherence theory, we have exposed the system-independent conditions under which the quantum decoherence dynamics is either well, or poorly, approximated by classical dynamics. Further studies are ongoing to assess QCC in cases beyond the short time and strong decoherence approximations. Preliminary computational results [8] support the conclusions drawn herein.

This work was supported by the U.S. Office of Naval Research and the Natural Sciences and Engineering Research Council of Canada. 
[1] W.H. Zurek, quant-ph/0105127; D. Giulini et al., Decoherence and the Appearance of a Classical World in Quantum Theory (Springer, New York, 1996).

[2] S. Habib, K. Shizume, and W.H. Zurek, Phys. Rev. Lett. 80, 4361 (1998); J. Gong and P. Brumer, Phys. Rev. E 60, 1643 (1999).

[3] For example, V. Milner et al., Phys. Rev. E 61, 7223 (2000); M.B. d'Arcy et al., Phys. Rev. E 64, 056233 (2001).

[4] For example, J. Wilkie and P. Brumer, Phys. Rev. A 55, 27 (1997); Phys. Rev. A 55, 43 (1997); C. Jaffé, S. Kanfer, and P. Brumer, Phys. Rev. Lett. 54, 8 (1985).

[5] M.A. Nielsen and I.L. Chuang, Quantum Computation and Quantum Information (Cambridge University Press, Cambridge, 2000).

[6] M. Shapiro and P. Brumer, Adv. Atom. Mol. and Opt. Phys., 42, 287 (2000).

[7] H. Wang et al., J. Chem. Phys. 114, 2562 (2001); F. Grossmann, J. Chem. Phys. 103, 3696 (1995); A.M.O. de Almeida, preprint quant-ph/0208094; V.S. Batista and P. Brumer, Phys. Rev. Lett. 89, 143201 (2002).

[8] H. Han, J. Gong, and P. Brumer, to be published.

[9] P.C. Lichtner and J.J. Griffin, Phys. Rev. Lett. 37, 1521 (1976); W.H. Zurek, S. Habib and J.P. Paz, Phys. Rev. Lett. 70, 1187 (1993); X-P. Jiang and P. Brumer, Chem. Phys. Lett. 208, 179 (1993).

[10] J.I. Kim et al., Phys. Rev. Lett. 77, 207 (1996).

[11] L.M. Duan and G.C. Guo, Phys. Rev. A 56, 4466 (1997); D. Bacon, D.A. Lidar, and K.B. Whaley, Phys. Rev. A 60, 1944 (1999).

[12] B.L. Hu, J.P. Paz, and Y. Zhang, Phys. Rev. D 47, 1576 (1993).

[13] R.P. Feynman and F.L. Vernon, Ann. Phys. (N.Y.) 24, 118 (1963); A.O. Calderia and A.J. Leggett, Ann. Phys. (N.Y.) 149, 374 (1983); B.L. Hu, J.P. Paz, and Y. Zhang, Phys. Rev. D 45, 2843 (1992).

[14] It is not surprising, in retrospect, that the classical and quantum results are in perfect agreement in the cases where the bath is harmonic, the coupling is linear or quadratic, and the system can be neglected. In this case it is expected that the quantum and classical Liouville dynamics are identical. What is important, however, is that this equivalence can be extended to the process of decoherence, a phenomenon assumed to be solely quantum in nature.

[15] C.C. Cheng and M.G. Raymer, Phys. Rev. Lett. 82, 4807 (1999); J.R. Anglin, J.P. Paz, and W.H. Zurek, Phys. Rev. A 55, 4041 (1997).

[16] D. Braun, F. Haake, and W.T. Strunz, Phys. Rev. Lett. 86, 2913 (2001).

[17] W.H. Zurek and J. P. Paz, Phys. Rev. Lett. 72, 2508 (1994); A. K. Pattanayak, Phys. Rev. Lett. 83, 4526 (1999); D. Monteoliva and J.P. Paz, Phys. Rev. Lett. 85, 3373 (2000). 